\title{
Is Adenotonsillar Size a Significant Factor on Voice in Children Undergoing Adenotonsillectomy?
}

\author{
Suleyman Emre Karakurt, Mehmet Ali Cetin, Ali Riza Yamur, Aykut Ikinciogullari, \\ Serdar Ensari and Haci Huseyin Dere
}

\begin{abstract}
Objective: To determine whether adenotonsillar size is a significant determinant of voice in children who have undergone adenotonsillectomy.

Study Design: Prospective cohort study.

Place and Duration of Study: Ear Nose Throat Clinic, Ankara Numune Training and Research Hospital, Ankara, Turkey, from July 2017 to June 2018.

Methodology: A total of 57 patients, who have been diagnosed with chronic adenotonsillitis and operated for obstruction or infection, were included in the study. Patients were divided into two groups according to their palatine tonsil sizes. Each patient performed voice analysis preoperatively and one month postoperatively, recruiting both objective and subjective methods. F0, jitter \% and shimmer \% values were assessed with objective methods; while subjective methods evaluated pediatric voice handicap index (pVHI) scores. Pre- and post-operative F0, jitter \% and shimmer \% values and pVHI scores from each study group were compared.

Results: In each study group, pre- and post-operative F0, jitter \% and shimmer \% values were found to be similar. In Group A, postoperative $\mathrm{pVHI}$ scores were found to be significantly reduced $(p<0.001)$. In Group $B$, however, pre- and postoperatively assessed pVHI scores were similar.

Conclusion: Adenotonsillar hypertrophy in children, who underwent adenotonsillectomy, seems to be an important and positively effecting factor on the subjective, but not the objective, parameters of voice.
\end{abstract}

Key Words: Voice, Voice handicap index, Acoustic, Tonsillectomy, Adenoidectomy, Palatine tonsil.

\section{INTRODUCTION}

Adenotonsillectomy is the most common surgical procedure performed by otolaryngologists and the second most commonly performed pediatric surgery. 1-3 Although adenotonsillectomy is often considered as a minor operation, it may lead to a number of major complications including haemorrhage, anaesthesia complications and velopharyngeal insufficiency. ${ }^{4-6}$ Considering the fact that this operation is performed mainly due to chronic tonsillitis and adenotonsillar hypertrophy, an accurate understanding of the risks associated with this operation by both the patient and the physician is of great importance.7,8 Despite advances in surgical and anaesthetic techniques, the morbidity associated with this procedure remains a major concern. ${ }^{9}$ One of the questions addressed to the clinician during the regular informational interviews about the mortality and morbidity with the patients and/or their parents is whether this operation may cause vocal changes. It is

Department of ENT, Ankara Numune Training and Research Hospital, Anakara, Turkey

Correspondence: Dr. Suleyman Emre Karakurt, ENT and HNS Clinic, Ankara Numune Training and Research Hospital,

Hacettepe Mahallesi Talatpasa Bulvari No. 44 Altindag,

Ankara, Turkey

E-mail: suleymanemrekarakurt@gmail.com

Received: September 24, 2018; Accepted: December 27, 2018 the responsibility of clinicians to respond to these specific concerns.

Alterations in vocal tract anatomy due to adenotonsillectomy are likely to lead to a change in resonance and in the voice as a consequence. Previous studies in the literature that have focused on the effects of adenotonsillectomy on voice have reported conflicting results. ${ }^{10-12}$ One possible explanation for this is that, those studies may have recruited children having adenotonsillar tissues with varying sizes.

As to authors' knowledge, the effects of adenotonsillar size on voice have not previously been centred on in detail by objective and subjective methods in children undergoing adenotonsillectomy, which was the objective of the present work.

\section{METHODOLOGY}

The study was approved by the local Ethics Committee. Subjects' parents were informed and their signed consents were taken. Children between 3-10 years, who had been diagnosed as chronic adenotonsillitis, and operated in this clinic for obstruction or infection between July 2017 and June 2018 were included in the study. Children with hoarseness, speech impairment, or any upper or lower respiratory tract infections were excluded. The subjects, who met the inclusion criteria were divided into two groups according to their palatine tonsil sizes. Palatine tonsillar hypertrophy was assessed 
according to the scale suggested by Brodisky. ${ }^{13}$ Pharyngeal tonsillar hypertrophy, on the other hand, was evaluated by direct inspection via nasal fiberoptic endoscopy. The patients with grade 3 or grade 4 palatine tonsillar hypertrophy with a pharyngeal tonsillar hypertrophy that obstructs more than $70 \%$ of the nasopharyngeal passage were included in Group A; the patients with grade 1 or grade 2 palatine tonsillar hypertrophy with a pharyngeal tonsillar hypertrophy that obstructs more than $70 \%$ of the nasopharyngeal passage were included in Group B. All patients underwent cold knife adenotonsillectomy under general anaesthesia.

The parents were asked to complete the Turkish pVHI questionnaire, which includes 23 questions, and evaluate the effects of the child's voice on his/her social, emotional and educational life and has previously proven to be a reliable and valid translation. The questionnaire was repeated one month after the operation. Each patient also underwent an acoustic voice analysis preand one-month postoperatively.

The $\mathrm{pVHI}$ questionnaire is a subjective pediatric voice evaluation tool which consists of questions for parents. Its main objective is to assess the effects of the child's voice on his/her daily life. $\mathrm{pVHI}$ includes 23 questions, concerning with the functional (7 questions), physical (9 questions) and emotional (7 questions) aspects. The parents score the questions on a scale of 5 points (i.e. 0 to 4). A higher total score is indicative of a more severe subjective voice disorder.

All patients were performed acoustic voice analysis using XION Medical DIVAS 2.5 Digital Voice Analysis software. Briefly, the subjects were asked to sit in front of a microphone (a USB Audio CODEC microphone plugged into a preamplifier) such that their mouth was located $30 \mathrm{~cm}$ away from the microphone. They were asked to articulate the letter "a" for at least 5 seconds. The procedure was repeated after 10 minutes. The obtained acoustic samples were saved on the computer using Creative Labs SB0240 Audigy 2 Platinum 6.1 sound card (Creative, Milpitas, California, USA) and Dr. Speech v.4 program (Tiger Electronics, Seattle,WA, USA), which runs on Windows XP operating system. Fundamental frequency (F0), jitter $\%$ and shimmer $\%$ measurements were run. Two measurements from each subject were averaged and recorded for further evaluation.

Pre- and post-operative F0, jitter \%, shimmer \% values and $\mathrm{pVHI}$ scores were compared. Postoperative changes in these parameters were assessed and intergroup comparisons were performed further to evaluate the effects of adenotonsil size on voice.

Quantitative data were expressed as mean with standart deviation or median with Interquartile Range. Qualitative data were expressed as frequency and percentage. The Shapiro-Wilk test was used to assess the distribution of data. Intergroup comparisons for independent variables were performed using Mann-Whitney U-tests. As for the dependent variables, the groups were compared with Wilcoxon signed-rank tests. $P<0.05$ values were deemed statistically significant. SPSS statistical software (SPSS for Windows version 21.0; SPSS Inc, Chicago, IL, USA) was used for all statistical calculations.

\section{RESULTS}

A total of 57 patients were included in this study, of whom, $26(45.6 \%)$ were included in group A and 31 $(54.4 \%)$ in group B. Group A had 13 boys $(50 \%)$ and 13 girls (50\%); while 20 boys $(64.5 \%)$ and 11 girls (35.5\%) were present in group $B$. The mean age of the patients in group $A$ and group $B$ were $6.15 \pm 2.03$ and $6.93 \pm 2.23$ years, respectively (Table I).

Overall, the median preoperative F0, jitter \%, shimmer $\%$ values were 212 (193-284), 0.58 (0.52-0.81), 1.4 (1.05-1.71), respectively. Postoperative measurements revealed these values as F0=225 (192-250), jitter\%= $0.55(0.41-0.81)$, shimmer $\%=1.38$ (0.97-1.9). Pre- vs. post-operative comparisons of these three parameters did not demonstrate any significant differences $(p=0.402$, $p=0.263$, and $p=0.159$, respectively). The median preand post-operative pVHI scores were 8 (3-16.5) and 6 (2-9.5), respectively, demonstrating a significant improvement one month after adeno-tonsillectomy $(p=0.001$, Table II).

In group A, pre- and post-operative measurements were as follows: $\mathrm{F} 0=217$ (203-292) and $236(197-256)$; jitter\%= $0.55(0.44-0.79)$ and $0.55(0.40-0.70)$; shimmer $\%=1.38$ (1.03-1.81) and 1.47 (0.88-1.92). Post-operative changes in these three parameters were found to be insignificant in this patient group $(p=0.620, p=0.416$ and $p=0.684$, respectively). In this group, the median pre- and postoperative pVHI scores were 14.5 (9.75-22) and 5 (1.75$8.5)$, respectively, demonstrating a significant improvement one month after adenotonsillectomy $(p<0.001$, Table III). In group A, subjective but not the objective parameters of voice seemed to improve after the operation.

Table I: Demographic characteristics of the patients included.

\begin{tabular}{l|c|c|c}
\hline & Group A & Group B & Total \\
\hline Boys & $13(50 \%)$ & $20(64.5 \%)$ & 33 \\
Girls & $13(50 \%)$ & $11(35.5 \%)$ & 24 \\
Age & $6.15 \pm 2.03$ & $6.93 \pm 2.23$ & $6.57 \pm 2.16$ \\
\hline
\end{tabular}

Table II: Statistical comparison of the pre- and post-operative F0, Jitter \%, Shimmer\% values and $\mathrm{pVHI}$ scores.

\begin{tabular}{l|c|c|c}
\hline & Group A & Group B & Total \\
\hline & Preoperative & Postoperative & p-value* \\
F0 & $212(91)$ & $225(58)$ & 0.402 \\
Jitter \% & $0.58(0.29)$ & $0.55(0.40)$ & 0.263 \\
Shimmer \% & $1.4(0.66)$ & $1.38(0.93)$ & 0.159 \\
pVHI & $8(13.5)$ & $6(7.5)$ & 0.001 \\
\hline
\end{tabular}

pVHI: Paediatric voice handicap index. ${ }^{*}$ Wilcoxon signed-rank test. 
Table III: Statistical comparison of the preoperative and postoperative F0, Jitter \%, Shimmer \% values and pVHI scores in group A and group B.

\begin{tabular}{|c|c|c|c|c|c|c|}
\hline & \multicolumn{3}{|c|}{ Group A } & \multicolumn{3}{|c|}{ Group B } \\
\hline & Preoperative & Postoperative & p-value* & Preoperative & Postoperative & $p$-value* \\
\hline F0 & 217 (89) & $236(59)$ & 0.620 & $205(73)$ & $221(68)$ & 0.394 \\
\hline Jitter \% & $0.55(0.35)$ & $0.55(0.30)$ & 0.416 & $0.62(028)$ & $0.55(0.54)$ & 0.339 \\
\hline Shimmer \% & $1.38(0.78)$ & $1.47(1.04)$ & 0.684 & $1.44(0.60)$ & $1.33(0.71)$ & 0.141 \\
\hline pVHI & $14.5(12.25)$ & $5(6.75)$ & $<0.001$ & $5(6)$ & $6(8)$ & 0.903 \\
\hline
\end{tabular}

pVHI: Paediatric voice handicap index.

${ }^{*}$ Wilcoxon signed-rank test.

In group $\mathrm{B}$, preoperative and postoperative values were as: F0=205 (190-263) and $221(180-248) ; j i t t e r \%=0.62$ $(0.54-0.82)$ and $0.55(0.46-1)$; shimmer\% $=1.44(1.1-1.7)$ and $1.33(1.1-1.81) ; \mathrm{pVHI}=5(2-8)$ and $6(2-10)$. None of these four parameters showed a significant postoperative improvement $(p=0.394, p=0.339, p=0.141$ and $p=0.903$, respectively, Table III). In group $B$, adenotonsillectomy did not lead to any improvement in either objective or subjective voice parameters.

Group A and B were found to be comparable with respect to the postoperative changes observed in the F0, jitter $\%$ and shimmer $\%$ values $(p=0.461, p=0.718$ and $p=0.724$, respectively). The only statistically significant improvement observed was in the mean $\mathrm{pVHI}$ scores of the patients in group $A(p<0.001)$.

\section{DISCUSSION}

Adenotonsillectomy did not seem to have any significant effects on either the subjective or the objective parameters of voice in the non-hypertrophic patient group (Group B) in this study. On the other hand, in the hypertrophic patient group (Group A), adenotonsillectomy has led to a significant improvement in the subjective parameters of voice; however, the objective parameters have remained unchanged.

F0 is the vibration rate of the vocal folds and it represents the resonance characteristics of the supralaryngeal vocal tract with regard to tongue articulation and placement. 10 Jitter is a useful measurement tool to detect the periodto-period perturbations. It reflects the inability of the vocal folds to support periodic vibration, and the presence of turbulence noise in voice signal. ${ }^{14}$ Shimmer is related to the period-to-period variability of voice. Lower jitter and shimmer values may indicate a better voice quality.

Voice is mainly produced in the larynx and it gains its acoustic characteristics inside the vocal tract. 15 Adenotonsillectomy does not directly affect the vocal folds, but it may alter the resonance of the vocal tract. Mora et al. reported that adenotonsillectomy operation significantly improved the F0, jitter and shimmer values in children with adenotonsillar hypertrophy. ${ }^{14}$ In contrast, a latter study by Dimatos et al., included the children with adenoid hypertrophy, demonstrating a grade 3 or 4 palatine tonsil hypertrophy (according to the Brodsky ${ }^{13}$ scale) and causing a more than $70 \%$ obstruction across the nasopharynx. It was reported that no significant change had been observed in the F0, jitter and shimmer values after adenotonsillectomy. ${ }^{16}$

There are a number of papers in the literature with conflicting results regarding the effects of adenotonsillectomy on voice, ${ }^{10-12}$ which may be attributable to the heterogeneity of the patient groups that had been studied, with respect to the degree of adenotonsillar hypertrophy. In other words, adenotonsillar size may be an important determinant of whether adenotonsillectomy will exert any effects on voice, or not. From this standpoint, this study focused on adenotonsillar size and the patient groups were established depending on the absence or presence of adenotonsillar hypertrophy. In both groups, the pre- and one-month postoperatively measured objective voice parameters (i.e. F0, jitter \% and shimmer \%) were statistically similar.

Salami et al. studied the effects of surgery in children undergoing tonsillectomy either alone or with adenoidectomy, and reported a significant improvement in $\mathrm{VHI}$ scores. ${ }^{10}$ Naraghi and colleagues similarly recruited $\mathrm{pVHI}$ in their study to evaluate the subjective voice parameters in children, most of whom had been operated for obstruction, and found a significant betterment in the post-adenotonsillectomy scores. ${ }^{17}$ They attributed this improvement to the amelioration of obstruction and rhinolalia. Nonetheless, the authors underscored the need for confirmation via further studies to be held on children being operated for infectious reasons. For subjective voice analysis, we have recruited the Turkish pVHI as proven by Özkan et al. to be a valid and reliable translation of the original $\mathrm{pVHI}$ test. ${ }^{18}$ It was first developed by Jacobson et al. and modified by Zur et al. ${ }^{19,20}$ In the non-hypertrophic patient group, no significant difference was found between the pre- and one-month postoperatively measured $\mathrm{pVHI}$ scores. On the other hand, the $\mathrm{pVHI}$ scores of the children in the hypertrophic patient group significantly improved after the operation.

\section{CONCLUSION}

Adenotonsillar size is not a significant determinant of postoperative betterment in the objective parameters of voice in children undergoing adenotonsillectomy. On the other hand, for cases with adenotonsillar hypertrophy, subjective parameters of voice may be expected to show 
some postoperative improvement. The children and their parents may be counselled accordingly under the light of these findings.

\section{REFERENCES}

1. Subramaniam V, Kumar P. Impact of tonsillectomy with or without adenoidectomy on the acoustic parameters of the voice. Arch Otolaryngol Head Neck Surg 2009; 135:966-9.

2. Kim YS, Hong SJ, Choi J, Lee SH, Kwon SY, Choi JH. Spontaneous tonsillar hemorrhage and post-tonsillectomy hemorrhage. Clin Exp Otorhinolaryngol 2010; 3:56-8.

3. Shapiro N. Tonsillectomy myths. AAO-HNS Bulletin 2005; 24:12-3.

4. Reilly MJ, Milmoe G, Pena M. Three extraordinary complications of adenotonsillectomy. Int $\mathrm{J}$ Pediatr Otorhinolaryngol 2006; 70:941-6.

5. Haapanen ML, Ignatius J, Rihkanen H, Ertama L. Velopharyngeal insufficiency following palatine tonsillectomy. Eur Arch Otorhinolaryngol 1994; 251:186-9.

6. Riaz A, Malik HS, Fazal N, Saeed M, Naeem S. Anaesthetic risks in children with obstructive sleep apnea syndrome undergoing adenotonsillectomy. J Coll Physicians Surg Pak 2009; 19:73-6.

7. Materia E, Di Domenicantonio R, Baglio G, Marchisio P, Perletti L, Lispi L, et al. Epidemiology of tonsillectomy and/or adenoidectomy in Italy. Pediatr Med Chir 2004; 26:179-86.

8. Brown PM. How safe is paediatric tonsillectomy? Int $\mathrm{J}$ Pediatr Otorhinolaryngol 2006; 70:575-7.

9. Buland K, Zahoor MU, Asghar A, Khan S, Zaid AY. Efficacy of single dose perioperative intravenous steroid (dexamethasone) for postoperative pain relief in tonsillectomy patients. $J$ Coll Physicians Surg Pak 2012; 22:349-52.

10. Salami A, Jankowska B, Dellepiane M, Crippa B, Mora R. The impact of tonsillectomy with or without adenoidectomy on speech and voice. Int J Pediatr Otorhinolaryngol 2008; 72:1377-84.
11. Liu X, Zheng Y, Tian P, Yang J, Zou H. The impact of tonsillectomy with or without adenoidectomy on voice: Acoustic and aerodynamic assessments. J Voice 2015; 29:346-8.

12. Li J, Yan Y, Wang L, Duan J, Zhang K. Study of voice changes pre- and post-tonsillectomy. Lin Chung Er Bi Yan HouTou Jing WaiKe Za Zhi 2012; 26:399-401.

13. Brodsky L, Poje CP. Tonsillitis, tonsillectomy and adenoidectomy. In: Bailey BJ, Johnson JT, Newlands SD, Eds. Head \& Neck Surgery-Otolaryngology, Baltimore: Lippincott Williams \& Wilkins, 2006: p.1184-98.

14. Mora R, Crippa B, Dellepiane M, Jankowska B. Effects of adenotonsillectomy on speech spectrum in children. Int $J$ Pediatr Otorhinolaryngol 2007; 71:1299-304.

15. Atan D, Apaydin E, Özcan KM, Ikinciogullari A, Çetin MA, Dere H. Does tonsillectomy affect voice in early or late postoperative periods in adults? J Voice 2017; 31:131.e5-131.e8.

16. Dimatos SC, Neves LR, Beltrame JM, Azevedo RR, Pignatari SS. Impact of adenotonsillectomy on vocal emission in children. Braz J Otorhinolaryngol 2016; 82:151-8.

17. Naraghi M, Adil S, Bastaninejad S, Dabiran S. Evaluation of pediatric voice handicap index and pediatric voice-related quality of life before and after adenotonsillectomy in pediatric population. IntJ Pediatr Otorhinolaryngol 2015; 79:388-91.

18. Özkan ET, Tüzüner A, Demirhan E, Topbas S. Reliability and validity of the Turkish pediatric voice handicap index. Int $J$ Pediatr Otorhinolaryngol 2015; 79:680-4.

19. Jacobson BH, Johnson A, Grywalski C, Silbergleit A, Jacobson G, Benninger MS, et al. The voice handicap index (VHI): Development and validation. Am J Speech Lang Pathol 1997; 6:66-70.

20. Zur KB, Cotton S, Kelchner L, Baker S, Weinrich B, Lee L. Pediatric voice handicap index (pVHI): A new tool for evaluating pediatric dysphonia. Int $J$ Pediatr Otorhinolaryngol $2007 ; 71: 77-82$. 